\title{
Transient Three-Dimensional Analysis of Nozzle Side Load in Regeneratively Cooled Engines
}

\author{
Ten-See Wang ${ }^{*}$ \\ NASA Marshall Space Flight Center, Huntsville, Alabama, 35812
}

\begin{abstract}
Three-dimensional numerical investigations on the start-up side load physics for a regeneratively cooled, high-aspect-ratio nozzle were performed. The objectives of this study are to identify the three-dimensional side load physics and to compute the associated aerodynamic side load using an anchored computational methodology. The computational methodology is based on an unstructured-grid, pressure-based computational fluid dynamics formulation, and a transient inlet condition based on an engine system simulation. Computations were performed for both the adiabatic and cooled walls in order to understand the effect of boundary conditions. Finite-rate chemistry was used throughout the study so that combustion effect is always included. The results show that three types of shock evolution are responsible for side loads: generation of combustion wave; transitions among free-shock separation, restricted-shock separation, and simultaneous free-shock and restricted shock separations; along with oscillation of shocks across the lip. Wall boundary conditions drastically affect the computed side load physics: the adiabatic nozzle prefers free-shock separation while the cooled nozzle favors restricted-shock separation, resulting in higher peak side load for the cooled nozzle than that of the adiabatic nozzle. By comparing the computed physics with those of test observations, it is concluded that cooled wall is a more realistic boundary condition, and the oscillation of the restricted-shock separation flow pattern across the lip along with its associated tangential shock motion are the dominant side load physics for a regeneratively cooled, high aspect-ratio rocket engine.
\end{abstract}

\section{Introduction}

$\mathrm{N}$ ozzle side loads are potentially detrimental to the integrity and life of almost all launch vehicle engines. For example, side load problems have been found for J2 engine ${ }^{1}$, Block-I Space Shuttle Main Engine (SSME), and recently, the Fastrac Engine. Most recently, the European Vulcain engine ${ }^{2}$ and the Japanese LE-7A engine ${ }^{3}$ have also experienced side load difficulties. A clear understanding of the mechanisms that contribute to side loads during engine startup, shutdown, and steady-state operations must be attained. Unfortunately, the current level of understanding nozzle side load physics is limited and does not allow the accurate prediction of side load severity for new nozzle designs. The lack of a prediction capability results in reduced life and increased weight for reusable nozzle systems. Simple models exist to predict side load magnitudes but are overly conservative and highly empirical. Subsequently, a detailed, predictive methodology based on computational fluid dynamics (CFD) is most promising in addressing these issues.

Wang ${ }^{4}$ reported the first attempt in using CFD to study transient flow separation inside an axisymmetric SSME nozzle, using scheduled start-up and shutdown sequences. Chen, et al ${ }^{5}$ reported a similar effort on an axisymmetric cold flow J2S nozzle later, using impulsive starts and cut-offs. Both captured the nozzle hysteresis phenomenon that is considered to be one of the basic characteristics of liquid rocket engine nozzles, and both fell short of predicting asymmetric flows due to the symmetric boundary condition limitation. Yonezawa, et al..$^{6}$ made the first twodimensional (2-D) nozzle side load prediction for the Japanese LE-7A engine. Yonezawa, et al. ${ }^{7}$ then made the first three-dimensional (3-D) side load prediction for the LE-7, LE-7A and CTP50-R5-L nozzles. They computed freeshock separation (FSS) and small side load for the LE-7, simultaneous occurrence of FSS and restricted-shock

"Technical Assistant, Thermal and Combustion Analysis Branch, ER43, Senior Member AIAA. 
separation (RSS) and medium side load for LE-7A, and transition from FSS to RSS, but missed three high side load peaks for the CTP50-R5-L nozzles. The authors stated that the grid used was probably too coarse, resulting in overestimated flow fluctuation and smearing of distinctive peaks.

After the works of Yonezawa, et $\mathrm{al}^{6,7}$, Wang ${ }^{8}$ performed a series of 2-D and axisymmetric numerical studies on the Block-I SSME start-up side load physics, using hybrid grids that were anchored for axial force and wall heat fluxes. ${ }^{9}$ The side load physics captured in that study include the Coanda effect, combustion wave, transitions between FSS and RSS, and shock oscillation across the lip. More importantly, it is found that inlet ramp rate and combustion drastically affect the computed side load physics. Note that Yonezawa, et al ${ }^{6,7}$ ignored combustion for LE-7 and LE-7A engines and assumed constant ramp rates. The lesson learned sets up the computational strategy for the 3-D investigation of the regeneratively cooled, Block-I SSME start-up side load physics in this study: a transient inlet condition based on an engine system simulation and the reacting flow calculation using finite-rate chemistry. Furthermore, the effect of wall cooling is investigated to see if wall boundary condition plays a role in side load physics.

\section{Computational Methodology}

\section{A. Computational Fluid Dynamics}

The CFD methodology is based on a multi-dimensional, finite-volume, viscous, chemically reacting, unstructured grid, and pressure-based formulation. Time-varying transport equations of continuity, momentum, total enthalpy, turbulence, and species continuity were solved using a time-marching sub-iteration scheme. A predictor and corrector solution algorithm was employed to provide coupling of the governing equations. A second-order central-difference scheme was employed to discretize the diffusion fluxes and source terms. For the convective terms, a second-order upwind total variation diminishing difference scheme was used. To enhance the temporal accuracy, a second-order backward difference scheme was employed to discretize the temporal terms. Details of the numerical algorithm can be found in Ref's 9-12.

An extended $k-\varepsilon$ turbulence model ${ }^{13}$ was used to describe the turbulence. A modified wall function approach was employed to provide wall boundary layer solutions that are less sensitive to the near-wall grid spacing. Consequently, the model has combined the advantages of both the integrated-to-the-wall approach and the conventional law-of-the-wall approach by incorporating a complete velocity profile and a universal temperature profile $^{14}$. A 7-species, 9-reaction detailed mechanism ${ }^{14}$ was used to describe the finite-rate, hydrogen/oxygen afterburning chemical kinetics. The seven species are $\mathrm{H}_{2}, \mathrm{O}_{2}, \mathrm{H}_{2} \mathrm{O}, \mathrm{O}, \mathrm{H}, \mathrm{OH}$, and $\mathrm{N}_{2}$.

\section{B. Simulated Start-up Sequence}

The thruster inlet properties were obtained from an engine system calculation, simulating the effect of valve sequences during a nominal $5 \mathrm{~s}$ operation. Figure 1 shows some of the inlet flow properties: the time-varying inlet pressure, temperature, and equivalence ratio profiles. Two significant pressure rise events can be identified. The first one occurs at $1.5 \mathrm{~s}$ due to oxygen prime, while the second one occurs at about $2.4 \mathrm{~s}$, caused by the step opening of the oxygen valves in the pre-burners. It can be seen that the thruster environment is fuel rich throughout the start-up transient, setting up the potential for afterburning. The high equivalence ratio in the first $0.2 \mathrm{~s}$ is not significant since the flow rate is negligible.

\section{Computational Grid Generation}

Parametric studies conducted ${ }^{9}$ show that a structured-cell dominated hybrid mesh performed more favorably than an unstructured-cell dominated hybrid mesh both in accuracy and efficiency, on flow physics and prediction of nozzle design parameters such as axial force and heat fluxes. Assuming that a grid suitable for axial force calculation is sufficient for side force calculation, the structured-cell dominated mesh was used in this study and its layout is shown in Fig. 2. The structured (hexahedral) cells are used in the thruster and plume region, while the unstructured (prismatic) elements are used in the freestream region. The grid was generated using a software package GRIDGEN. ${ }^{15}$ The total number of points is $1,286,934$, comparing to the $145,500,145,500$, and 405,900 points used on LE-7, LE-7A, and CTP50-R5-L, ${ }^{7}$ respectively. 


\section{Boundary and Inlet Conditions}

Fixed total condition was used for the outer boundary and a total pressure of $1 \mathrm{~atm}$ was used to simulate the nozzle hot-firing at sea level. No-slip condition was specified for the solid walls. The inlet flow properties obtained from the system simulation include the time varying total pressure, temperature, and propellant composition. The time varying propellant composition was preprocessed with the Chemical Equilibrium Calculation program ${ }^{16}$, assuming the propellants were ignited to reach equilibrium composition immediately beyond the injector faceplate. The fuel rich environment indicates the inlet composition contains mostly steam and excess hydrogen.

\section{Results and Discussion}

The computations were performed on several cluster machines using $22 \sim 32$ processors. For the nominal $5 \mathrm{~s}$ start-up sequence, global time steps of $10 \mu$ s were used in the beginning, reduced to $2.5 \mu$ s during the combustion wave event, increased to $5 \mu \mathrm{s}$ afterwards, then reduced to $1 \sim 2.5 \mu$ s between the $3 \sim 3.1 \mathrm{~s}$ time frame, and resumed to $5 \mu \mathrm{s}$ the rest of the way. The adiabatic wall case was computed first; as the elapsed time reached $1.5 \mathrm{~s}$, the cooled wall case was started in parallel by specifying a cooled wall temperature distribution obtained from a separate conjugate heat transfer calculation. This procedure assumes the flow processes were identical for the two cases prior to $1.5 \mathrm{~s}$. The computed side forces for the adiabatic and cooled wall cases from $0 \sim 2 \mathrm{~s}$ are shown in Fig's 3 and 4 , respectively, covering the first pressure rise event. It can be seen that the overall computed side load for the adiabatic nozzle is higher than that of the cooled nozzle for the first $2 \mathrm{~s}$.

As indicated in Fig. 1, the pressure rises rather slowly, after the start command. Yet the chamber gradually fills up with steam and hydrogen and a detached core jet emerges from the throat at around $0.175 \mathrm{~s}$. Unlike the Coanda effect ${ }^{17}$ captured in the low aspect-ratio, 2-D planar nozzle, ${ }^{8}$ with which the core jet and subsequent Mach disk flow adhere to the wall, creating asymmetric flow and producing early side forces; the three-dimensional core jet is fairly centered, however, and produces negligible side forces during the core jet flow ${ }^{8}$ period (Fig's 3 and 4). As a result, even as the Mach disk flow ${ }^{8}$ develops after $1.2 \mathrm{~s}$ and some small side load appears between 1.2 and $1.5 \mathrm{~s}$, the flow is still fairly centered. It is speculated that the geometrical volume available for air pumping between the core jet and the wall is much larger in a 3-D nozzle than that of a 2-D nozzle, hence the difference. The side load produced in that period is believed to be caused by the oscillation of the supersonic jets of the Mach disk flow, which is in a FSS mode since the supersonic jets are oscillating freely and away from the wall. As time goes on, the magnitude of the oscillation increases because the pressure increases.

\section{A. Combustion Wave}

At $1.5 \mathrm{~s}$ into the start-up transient, the composition of the Mach disk flow is essentially hydrogen-rich steam. That leads to afterburning in the mixing layer between the supersonic jet and the ambient air, raising temperature, as shown in the temperature contours at $1.503 \mathrm{~s}$ in Fig. 5. That elevated temperature front quickly spreads through the mixing layer, creating a fast expanding hot gas that glows, much like that observed in the hot-fire test, as indicated in the rest of the plots in Fig. 5. The combustion wave starts at the mixing layer inside the nozzle and propagates in all directions. A portion of the combustion wave moving inside the nozzle would hit the wall, generating the first significant side load (Fig's 3 and 4) and several smaller ones thereafter due to shock reflections, while the rest expands away, as depicted in the pressure contours shown in Fig. 6. Since this combustion wave happens in the mixing layer for both adiabatic and cooled nozzle cases and not affected by the wall temperature, only the contours for the cooled nozzle are shown.

\section{B. Shock Transitions}

Soon after the disappearance of the combustion wave, the partially depleted hydrogen-rich jet gradually recovers and the free-shock separated Mach disk flow propagates again. Since the afterburning continues in the mixing layer, adding instability to the already unstable supersonic jet, the Mach disk itself oscillates back and forth, not only responding to the oscillating supersonic jets, but also adjusting to the changes in nozzle contour and the strengthening upstream flows. For the cooled nozzle, Fig. 7 shows a typical oscillating, free-shock separated Mach disk flow at $1.515 \mathrm{~s}$ where the supersonic jet stemming from the Mach disk steers away from the wall. At $1.520 \mathrm{~s}$, however, the supersonic jet moves toward the wall - an obvious transiting stage due to the Coanda effect. ${ }^{8.17} \mathrm{At}$ $1.525 \mathrm{~s}$, the supersonic jet completely adheres to the wall, enclosing a recirculation region, resulting in a distinctive RSS pattern. At $1.530 \mathrm{~s}$, the disk oscillates and retreats upstream. Note the supersonic jets are asymmetric at 1.525 and $1.530 \mathrm{~s}$. The RSS flow pattern persists thereafter, as indicated in Fig. 4. The transition from FSS to RSS creates the second significant side load, as shown in Fig's 3 and 4. 
For the adiabatic nozzle, although FSS-to-RSS also occurs at $1.520 \mathrm{~s}$, the RSS only lasts from 1.521 to $1.523 \mathrm{~s}$, after which RSS-to-FSS occurs (at $1.524 \mathrm{~s}$ ) and it is FSS thereafter, as indicated in Fig. 3. This is the first sign of difference in computed physics between the adiabatic and cooled nozzles. That is, after the first FSS-to-RSS transition, the adiabatic nozzle prefers FSS while the cooled nozzle favors RSS. This phenomenon may be explained by the boosted Coanda effect due to cooled wall. With a cooled wall, density is higher in the wall boundary layer. That leads to higher eddy viscosity, higher momentum, thinner boundary layer and lower pressure. Coanda effect then takes over, drawing the supersonic jet to the wall. For an adiabatic wall where the temperature is stagnation temperature, the opposite is true. This cooled wall flow separation physics agree with those reported in Ref's 18 and 19 in which thinner, cold-wall boundary was found to be less susceptible to separation than were the hot-wall, in steady nozzle flows. The RSS flow pattern is considered as mostly attached because the supersonic jet is reattached to the wall after the reattachment point. Note that the adiabatic nozzle flow is also more energetic, thus the associated FSS flow pattern is less stable with which the supersonic jet flops, attempting to become attached, and resulting in several jumps in side load in Fig. 3, from 1.6 to $2 \mathrm{~s}$.

Between 2 and $2.875 \mathrm{~s}$, the exhausting FSS of the adiabatic nozzle still flops, while the exhausting RSS of the cooled nozzle remains relatively symmetric, as seen in the side load profiles in Fig's 8 and 9, respectively. After the second pressure rise event at $2.4 \mathrm{~s}$, the flopping of FSS of the adiabatic nozzle becomes even stronger and the supersonic jet finally attaches to the wall, forming the simultaneous FSS and RSS, or partial RSS (or FSS) flow pattern. That creates FSS $\leftrightarrow 1 / 4$ RSS transition between 2.425 and $2.8 \mathrm{~s}$, as shown in Fig. 8. The "1/4 RSS" means the RSS covering about a quarter of the separation region circumferentially, while the rest of the region is associated with the FSS. Unlike the FSS-to-RSS transition described in Fig's 3 and 4, the FSS $\leftrightarrow 1 / 4$ RSS transition represents many a back-and-forth transitions between FSS and $1 / 4$ RSS. Figure 10 shows a snapshot of a typical $1 / 4$ RSS flow pattern in which the y-plane Mach number contours show a FSS, but the z-plane contours indicate an attached supersonic jet in the lower portion of the Mach disk flow, at $2.625 \mathrm{~s}$. Note that the Mach stem of the RSS is longer than that of the FSS in the z-plane, showing flow asymmetry and creating higher side loads. When the separation line on the wall is plotted, the result is mostly a slanted plane, as indicated in the wall $\mathrm{OH}$ concentration contours in Fig. 10. This slanted plane is very similar to the "tilted plane" described in Ref. 1, except the underlying physics computed in here is much more complicated because of the partial RSS flow pattern. The attached supersonic jet and the recirculation region impress a "hot region" beneath the lowest point of the slanted plane.

Figure 11 shows the RSS flow patterns of the cooled nozzle at $2.580 \mathrm{~s}$. It can be seen from the Mach number contours that the RSS flow pattern is more symmetric than that of the partial RSS flow pattern shown in Fig. 10. This is further supported by the wall $\mathrm{OH}$ concentration contours appearing as an almost symmetric band. That $\mathrm{OH}$ "band" represents the contact surface impressed by the recirculated region, which is bounded by the two stems of the Lambda shock inside which are combustion products. Note the Mach disk on the y-plane slants to the right, while that on the z-plane slants to the left, indicating the asymmetric nature of the relatively symmetric restricted-shock separated Mach disk flow. Figures 10 and 11 represent typical flow patterns of adiabatic and cooled nozzles, from 2.425 to $2.8 \mathrm{~s}$ and from $2 \sim 2.8 \mathrm{~s}$, respectively.

\section{Shock Oscillations across the Lip}

Fơ the adiabatic nôzżle, the shock transition activity culminates betwecen 2.8 and $3.04 \mathrm{~s}$, gencrating the highcst side load during its second pressure rise event. This is because the random transitions from FSS to partial RSS occur. E.g., we may have FSS-to-3/4 RSS first and then FSS-to-1/4 RSS next. Afterwards, the mostly FSS flow pattern oscillates in and out of the lip from 3.04 to $3.22 \mathrm{~s}$, generating side loads which are lower than those due to FSS to partial RSS transitions. Occasionally, the " $1 / 4$ RSS" pattern appears in that time period. With the cooled nozzle, after a period of nonchalant oscillations between 2 and $2.875 \mathrm{~s}$, the RSS flow pattern moves in-and-out of the lip repeatedly, until $3.15 \mathrm{~s}$, generating maximum side load doubling that of the adiabatic nozzle, as shown in Fig. 9. The mostly symmetric two-stemmed RSS flow pattern goes asymmetric while crossing the nozzle lip, especially during the receding portion of the oscillation when the shock moves tangentially. The disturbance of lip-crossing caused by the two-stemmed RSS is stronger than that of one-stemmed FSS, especially near the lip for a high aspectratio nozzle where the circumferential surface area is largest.

Fig. 12 shows the computed side force loci for the adiabatic nozzle in four consecutive installments, covering 2.4 to $3.3575 \mathrm{~s}$. There is no obvious tangential motion, except for a very short period from 3.008125 to $3.025 \mathrm{~s}$ during which occasional RSS appears. For the cooled nozzle, large tangential motion is observed frequently during the lip RSS oscillation period, as shown in the four representative tangential force loci in Fig. 13. It is also noticed that the side force vector moves counter-clockwise (looking into the nozzle) during the tangential motion of the receding, and not the exhausting part of the shock oscillation. Moreover, the characteristics of the four snapshots in Fig. 13 and those in Fig. 12 signify the relationship among RSS, tangential motion and larger side force. Since the tangential 
shock motions were observed in both the SSME and J2S hot-firings, it is deduced that physics computed with cooled wall boundary condition are more realistic for the regeneratively cooled engines.

Fig. 14 and 15 show the selected exhausting and receding wall $\mathrm{OH}$ contours of the adiabatic and cooled nozzle, respectively. In Fig. 14, at $2.83 \mathrm{~s}$, the wall OH contours again represent the separation line of an exhausting FSS, and it transits into that of a simultaneous FSS and $1 / 2$ RSS at $2.84 \mathrm{~s}$. The RSS portion moves faster than that of the FSS, hence a "valley" forms and reaches the lip at $2.845 \mathrm{~s}$. The exhausting motion then turns into a receding motion while the RSS portion diminishing at $2.8475 \mathrm{~s}$. In Fig. 15, at $2.92625 \mathrm{~s}$, the slanted wall $\mathrm{OH}$ contours near the lip represent an exhausting RSS separation band. About half of that band would leave the nozzle at $2.9275 \mathrm{~s}$. Note the asymmetry of that band. The band disappears completely at $2.9325 \mathrm{~s}$ while the nozzle is flowing full. At $2.9375 \mathrm{~s}$, the recovering RSS separation band returns, completing one of the many cycles of RSS oscillation across the lip. Note again the asymmetry of the band.

Fig. 16 shows the computed wall pressure, shear stress, and heat flux histories for the cooled nozzle at a monitoring point near the lip. Those at the same axial location but circumferentially away from this point by 90 , 180 , and 270 degrees are similar to these and not shown for clarity. The fluctuation of these properties is caused by the RSS oscillation across the lip. These profiles are qualitatively similar as expected. More importantly, the fact that the heat flux history appears to correlate with that of pressure and shear stress signifies the transient thermal load may also play a role in the side load physics. In fact, Watanabe, et al. reported ${ }^{20}$ damage to some regenerative cooling tubes by strong heat-load during the start-up and shutdown processes in early LE-7A engine development. Fourier analyses were performed for pressure and heat flux histories between $2.90375 \sim 3.0225 \mathrm{~s}$ for all four monitoring points. The result is shown in the power spectral density profiles in Fig. 17. The dominant frequency based on the pressure is about $122 \mathrm{~Hz}$, while that based on heat flux is nearly the same at about $125 \mathrm{~Hz}$ (see Table 1 ), indicating the wall heat flux is approximately synchronized with that of pressure.

The same Fourier analyses were performed with computed wall pressure and temperature histories for the adiabatic nozzle between 3.04 and $3.2225 \mathrm{~s}$, during which the fluctuations are caused by the FSS oscillation across the lip. The dominant frequency based on pressure is about $45 \mathrm{~Hz}$, while that based on temperature is slightly higher at about $49 \mathrm{~Hz}$, as shown in Table 1. The lower dominant frequency of FSS oscillation appears to correlate with lower side load, while the higher dominant frequency of RSS oscillation appears to correlate with higher side load.

Table 1. Computed dominant frequencies.

\begin{tabular}{|c|c|c|}
\hline & Dominant frequency, $\mathrm{Hz}$ & Variable \\
\hline Adiabatic nozzle & 45 & pressure \\
\cline { 2 - 3 } & 49 & temperature \\
\hline \multirow{2}{*}{ Cooled nozzle } & 122 & pressure \\
\cline { 2 - 3 } & 125 & Heat flux \\
\hline
\end{tabular}

Table 2 shows a comparison of the computed and measured peak side loads. The measured side load profile comprises of two peaks or two modes. The first peak occurs right after the first pressure-rise event or $1.5 \mathrm{~s}$ (see Fig's 3 and 4), while the second peak or the maximum side load happens well after the second pressure-rise event or $2.4 \mathrm{~s}$ (see Fig's 8 and 9). It can be seen that the combustion wave induced side load was not measured. This is because precautionary measures such as sparklers (not simulated in this study) were placed near the nozzle exit to burn off excess fuel such that potentially detrimental situation is avoided. The role of combustion wave induced side load physics is hence considered less important since sparklers will always be used. The computed first mode peak side loads for both boundary conditions agree reasonably well with that of measurement. As for the computed $2^{\text {nd }}$ mode side loads, those associated with the adiabatic nozzle appear too low, while that associated with the cooled nozzle compares reasonably well with the measurement.

Table 2. A comparison of peak side loads.

\begin{tabular}{|c|c|c|c|l|}
\hline $\mathrm{F}_{\mathrm{yz}}, \mathrm{kN}$ & Test & Adiabatic nozzle & Cooled nozzle & Computed side load physics \\
\hline- & - & 395 & 176 & Combustion wave \\
\hline $1^{\text {st }}$ mode & \multirow{2}{*}{$0^{*}$} & 70 & 80 & First FSS-to-RSS \\
\cline { 3 - 5 } & & 102 & - & Following RSS-to-FSS \\
\hline \multirow{2}{*}{$2^{\text {nd }}$ mode } & \multirow{2}{*}{$200^{*}$} & 110 & - & FSS-to-partial RSS \\
\cline { 3 - 5 } & & 60 & - & FSS oscillation across lip \\
\cline { 3 - 6 } & & - & 212 & RSS oscillation across lip \\
\hline
\end{tabular}


In summary, contrary to the intuition or results ${ }^{9}$ of axial force computation that cooled wall boundary condition gives lower thrust than that of adiabatic wall due to energy loss, the cooled nozzle produces much higher peak side force than that of adiabatic nozzle, because the RSS oscillation at the lip is much more energetic than either that of the FSS or the FSS-to-partial RSS transitions. Moreover, the cooled wall boundary condition appears to give more realistic side load physics than those of the adiabatic wall, leading to the conclusion that the first side load mode is generated by the FSS-to-RSS transition, while the dominant second side load mode is caused by the RSS oscillation across the lip along with its associated tangential shock motion.

\section{Conclusions}

Three-dimensional numerical investigations on the Block-I SSME start-up side load physics and predictions of the associated aerodynamic side load for adiabatic and cooled nozzles were performed. The results show that three types of shock evolutions generate significant side loads: the occurrence of combustion wave, transitions among FSS, RSS, and simultaneous FSS and RSS, and shock oscillations across the lip. The results also show that boundary conditions affect the computed side load physics. The creation of the combustion wave and the first transition from FSS to RSS are common to both adiabatic and cooled nozzles just after the first pressure rise event. After which the adiabatic nozzle prefers the FSS, while the cooled nozzle favors the RSS. After the second pressure rise event, the peak side load of the adiabatic nozzle occurs due to the numerous transitions between FSS and partial RSS, after which lip FSS oscillation follows until the nozzle flows full; for the cooled nozzle, RSS persists throughout, and significant side load happens during the RSS oscillation across the lip. By comparing the computed results with those of test observations, it is deduced that cooled wall is a more realistic boundary condition than that of an adiabatic wall for a regeneratively cooled engine. In addition, since the side load induced by combustion wave may be avoided with sparklers, and that induced by the FSS-to-RSS transition is considerably lower, the RSS oscillation across the lip along with its associated tangential shock motion appear to be the dominant side load physics for the regeneratively cooled, high aspect-ratio rocket engines.

\section{Acknowledgments}

This study was partially supported by a MSFC FY03 CDDF effort entitled "Nozzle Side Load Technology". The author wishes to thank Chief Aerodynamicist Werner Dahm for his guidance, and Dave Seymour for engine start-up simulation. Y.-S. Chen of Engineering Sciences, Inc. provided technical support and discussions. James Beck of Rocketdyne Boeing provided test observations on SSME side load physics and suggested cooled wall computation. Discussions with Joe Ruf, Daniel Dorney, and Kader Frendi of University of Alabama at Huntsville are also acknowledged.

\section{References} i⿳⺈⿴囗十.

${ }^{1}$ Nave, L.H., and Coffey, G.A., "Sea Level Side Loads in High-Area-Ratio Rocket Engines," AIAA Paper 73-1284, Nov.

${ }^{2}$ Hageman, G., Alting, J., and Preclik, D., "Scalability for Rocket Nozzle Flows Based on Subscale and Full-Scale Testing," Journal of Propulsion and Power, Vol. 19, No. 3, 2003, pp.321-331.

${ }^{3}$ Wantabe, Y., Sakazume, N., and Tsuboi, M., "LE-7A Engine Nozzle Problems During the Transient Operations," AIAA Paper 2002-3841, July 2002.

${ }^{4}$ Wang, T.-S., "Numerical Study of the Transient Nozzle Flow Separation of Liquid Rocket Engines," Computational Fluid Dynamics Journal, Vol. 1, No. 3, 1992, pp. 319-328.

${ }^{5}$ Chen, C.L., Chakravathy, S.R., and Hung, C.M., "Numerical Investigation of Separated Flows," AIAA Journal, Vol. 32, No. 9, 1994, pp. 1836-1843.

${ }^{6}$ Yonezawa, K., Yokota, K., Watanabe, Y., Tsujimoto, K., and Abe, T., "2-D Numerical Simulation of Side Loads in Rocket Nozzles," Proceedings of $23^{\text {rd }}$ International Symposium on Space Technology and Science, Japan Society for Aeronautical and Space Sciences, 2002, pp. 1146-1151.

${ }^{7}$ Yonezawa, K., Yokota, K., Tsujimoto, K., Sakazume, N., and Watanabe, Y., "Three-Dimensional Unsteady Flow Simulation of Compressed Truncated Perfect nozzles," AIAA Paper 2002-3991, July 2002.

${ }^{8}$ Wang, T.-S., "Transient Two-Dimensional Analysis of Side Load in Liquid Rocket Engine Nozzles," AIAA Paper 20043680, 11-14 July, 2004.

${ }^{9}$ Wang, T.-S., "Multidimensional Unstructured-Grid Liquid Rocket Engine Nozzle Performance and Heat Transfer Analysis," AIAA Paper 2004-4016, 11-14 July, 2004.

${ }^{10}$ Chen, Y.-S., Liu, J., Zhang, S., and Mallapragada, P., "An Integrated Tool for Launch Vehicle Base-Heating Analysis," Final Report, Engineering Sciences, Inc., Huntsville, AL, December 2001. 
${ }^{11}$ Wang, T.-S., Chen, Y.-S., Liu, J., Myrabo, L.N., and Mead, F.B. Jr., "Advanced Performance Modeling of Experimental Laser Lightcraft," Journal of Propulsion and Power, Vol. 18, No. 6, 2002, pp. 1129-1138.

${ }^{12}$ Chen. Y.-S., Zhang S., and Liu, J., "Stage Separation Performance Analysis Project," Final Report, Engineering Sciences, Inc., Huntsville, AL, June, 2002.

${ }^{13}$ Chen, Y.-S., and Kim, S. W., "Computation of Turbulent Flows Using an Extended k- $\varepsilon$ Turbulence Closure Model," NASA CR-179204, Oct. 1987.

${ }^{14}$ Wang, T.-S., Droege, A., D’Agostino, M., Lee, Y.-C., and Williams, R., “Asymmetric Base-Bleed Effect on X-33 Aerospike Plume Induced Base-Heating Environment," Journal of Propulsion and Power, Vol. 20, No. 3, 2004, pp. 385-393.

${ }^{15}$ Steinbrenner, J.P., Chawner, J.R., and Fouts, C., "Multiple Block Grid Generation in the interactive Environment," AIAA Paper 90-1602, June 1990.

${ }^{16}$ Svehla, R.A., and McBride, B.J., "FORTRAN IV Computer Program for Calculation of Thermodynamic and Transport Properties of Complex Chemical Systems," NASA TN D-7056, Jan. 1973.

${ }^{17}$ Kumada, M., Mabuchi, I., and Oyakawa, K., "Studies on Heat Transfer to Turbulent Jets with Adjacent Boundaries," Bulletin of the Japan Society of Mechanical Engineers, Vol. 16, No. 100, 1973, pp. 1712-1722.

${ }^{18}$ Chang, C.L., Kronzon, Y., and Merkle, C.L., "Time-Iterative Solutions of Viscous Supersonic Nozzle Flows," AIAA Journal, Vol. 26, No. 10, 1988, pp. 1208-1215.

${ }^{19}$ Shimura K., Asako, Y., and Lee, J.H., "Numerical Analysis for Supersonic Flows in a Cooled Nozzle," Numerical Heat Transfer, Part A., Vol. 26, 1994, pp.631-641.

${ }^{20}$ Watanabe, Y., Sakazume, N., Yonezawa, K., and Tsujimoto, Y., "LE-7A Engine Nozzle Flow Separation Phenomenon and the Possibility of RSS Suppression by the Step inside the Nozzle," AIAA Paper 2004-4014, 11-14 July, 2004. 

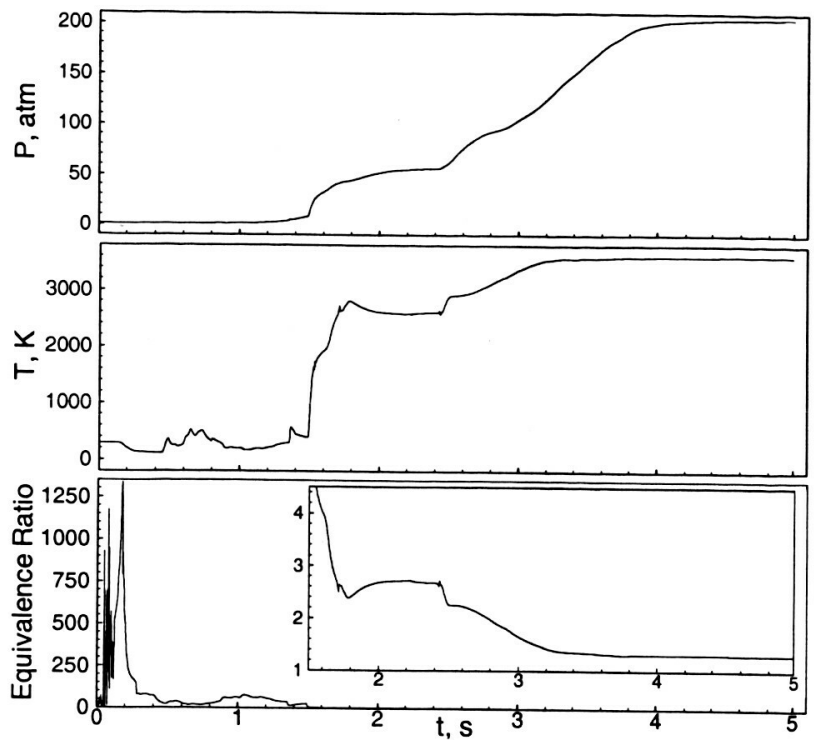

Fig. 1 Simulated thruster inlet properties during the startup transient.

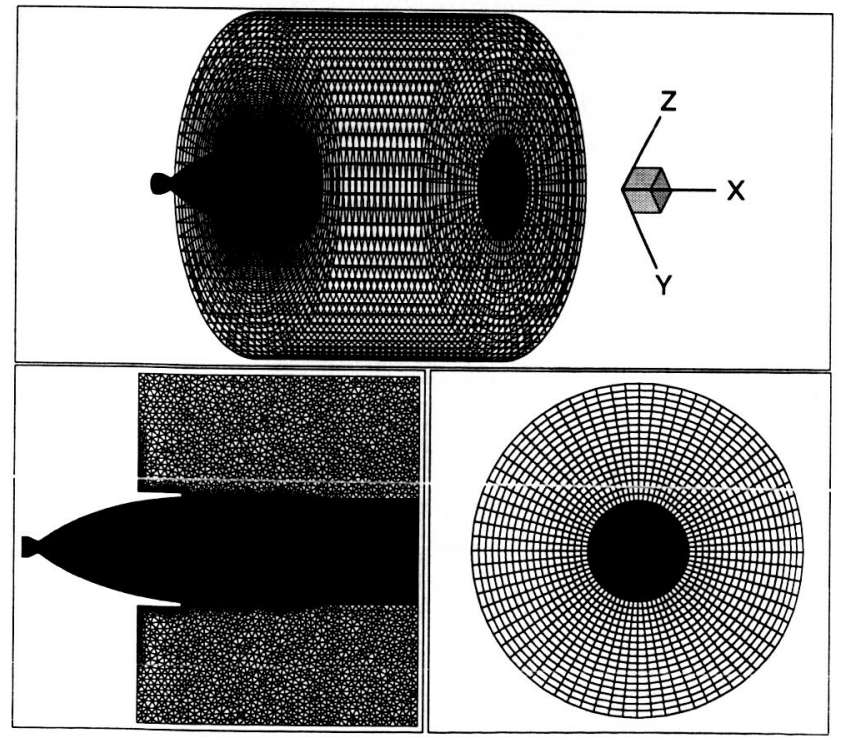

Fig. 2 The layout of hybrid grid 3d6. Top: an overall view. Bottom left: a cross-sectional cut through the nozzle axis. Bottom right: the exit plane.

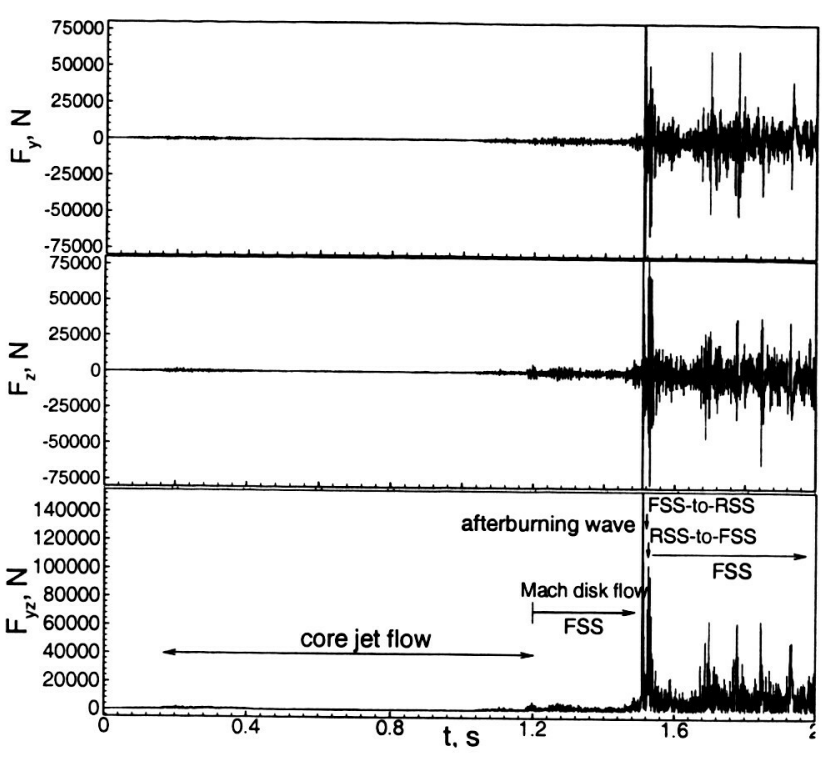

Fig. 3 Computed side forces for the adiabatic nozzle from $0 \sim 2 \mathrm{~s}$.

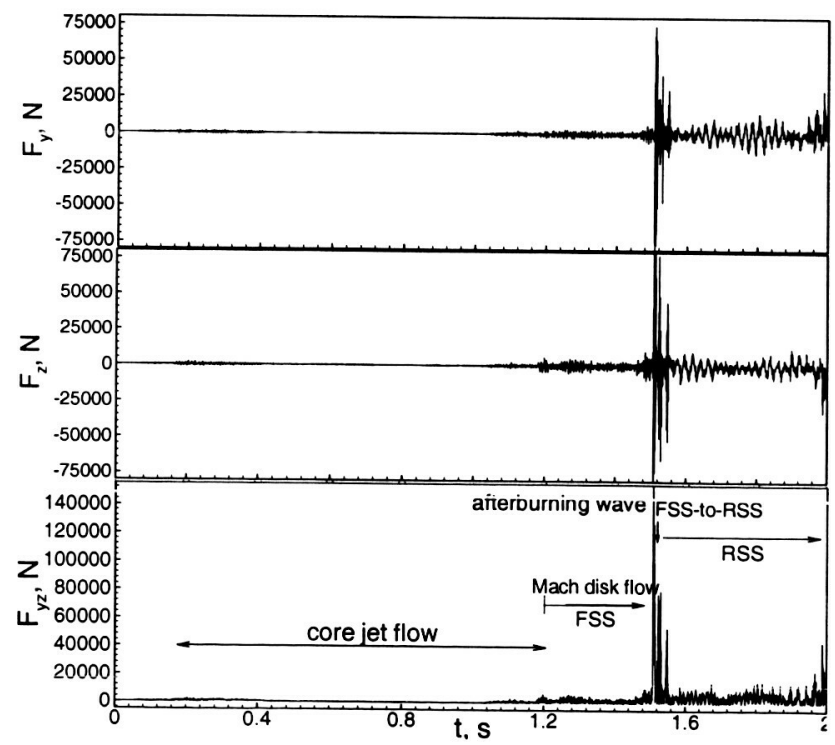

Fig. 4 Computed side forces for the cooled nozzle from 0 $\sim 2 \mathrm{~s}$. 

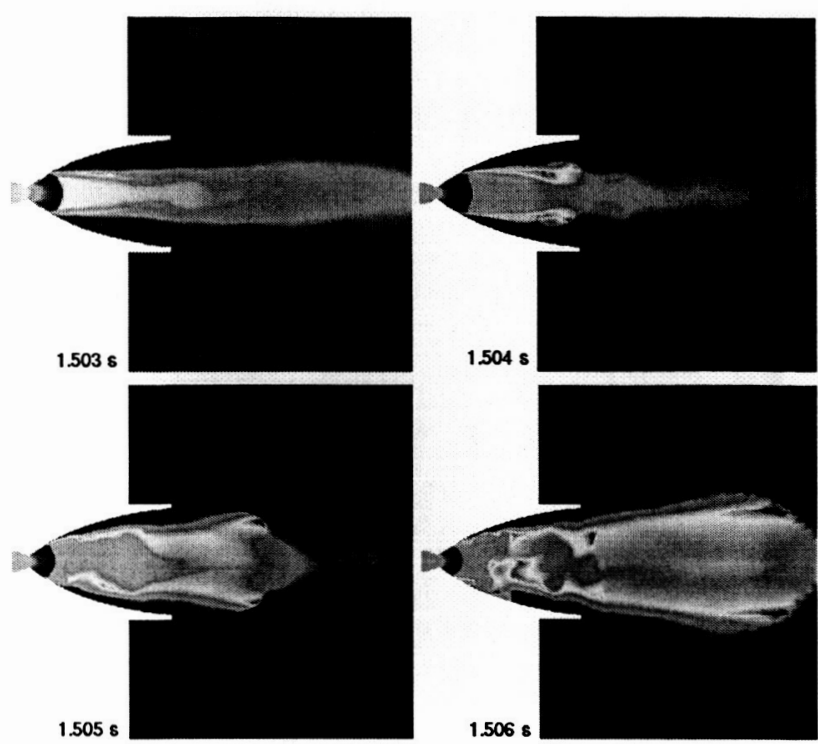

Fig. 5 Computed y-plane temperature contours of the cooled nozzle showing combustion wave.
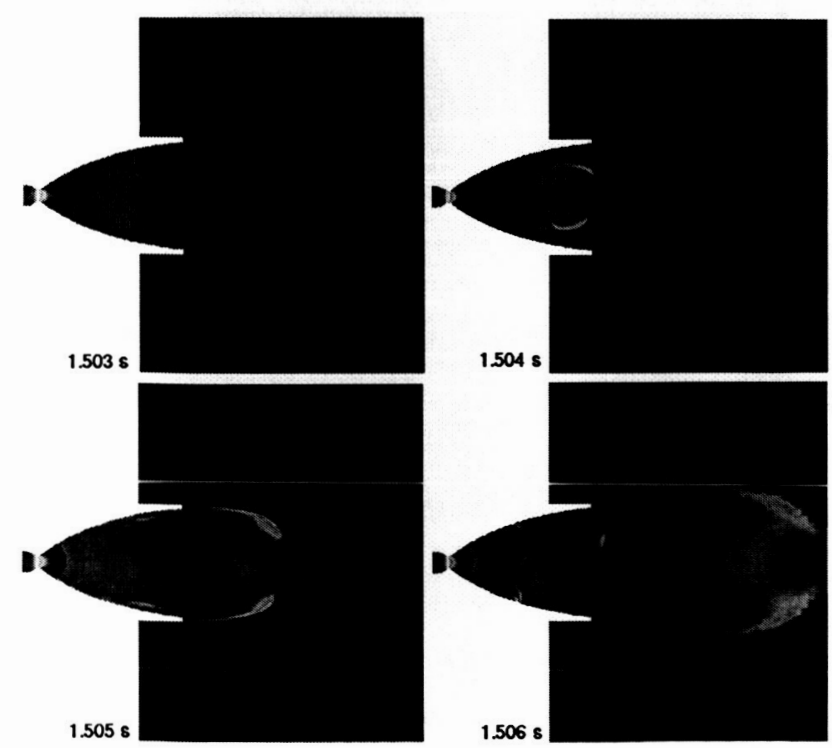

Fig. 6 Computed y-plane pressure contours of the cooled nozzle showing combustion wave.

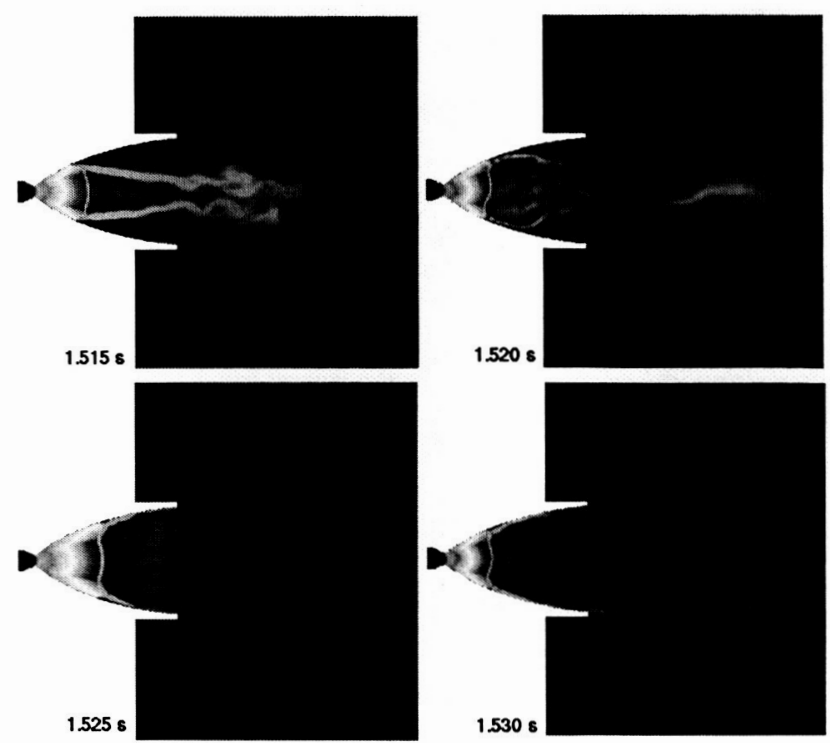

Fig. 7 Computed z-plane Mach numbers contours of the cooled nozzle representing FSS-to-RSS transition.

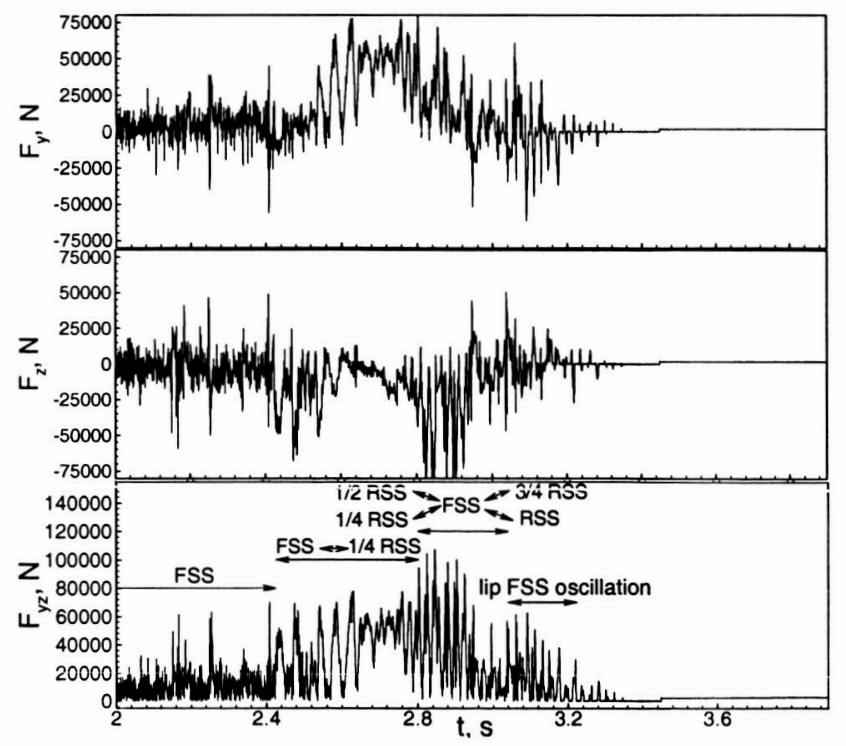

Fig. 8 Computed side forces for the adiabatic nozzle from $2 \sim 4 \mathrm{~s}$. 


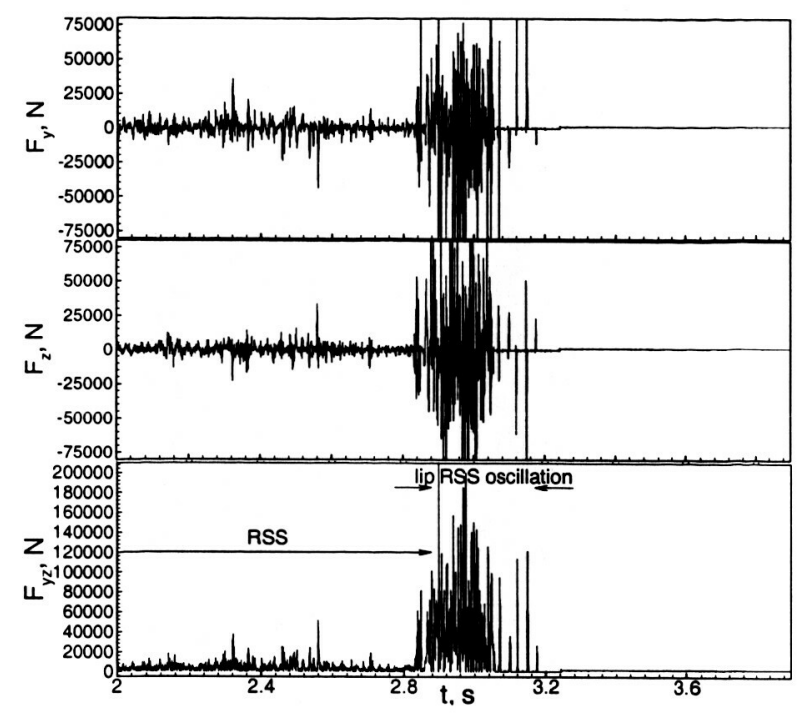

Fig. 9 Computed side forces for the cooled nozzle from $2 \sim 4 \mathrm{~s}$.
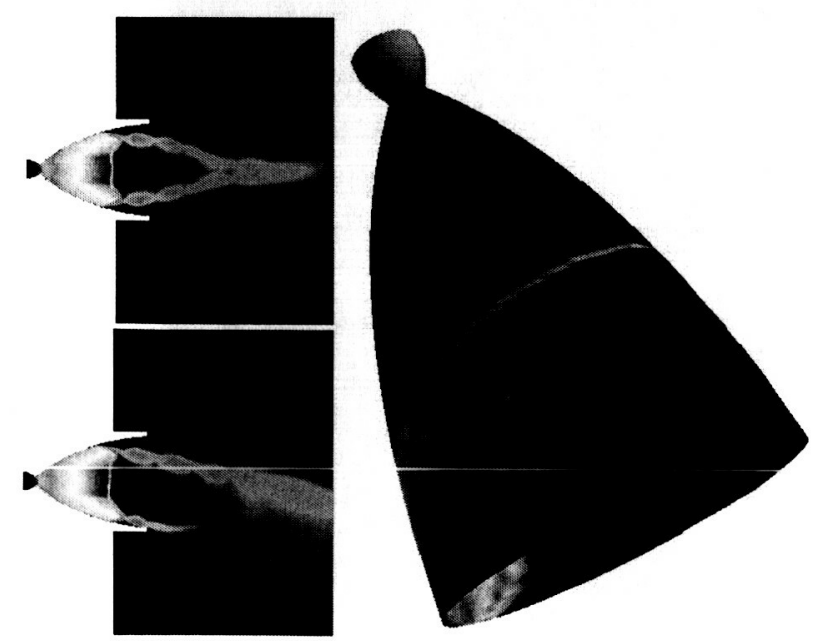

Fig. 10 Computed scalar contours for the adiabatic nozzle at $2.625 \mathrm{~s}$. Top left: Mach number contours on yplane. Bottom left: Mach number contours on z-plane. Right: wall $\mathrm{OH}$ concentration contours.

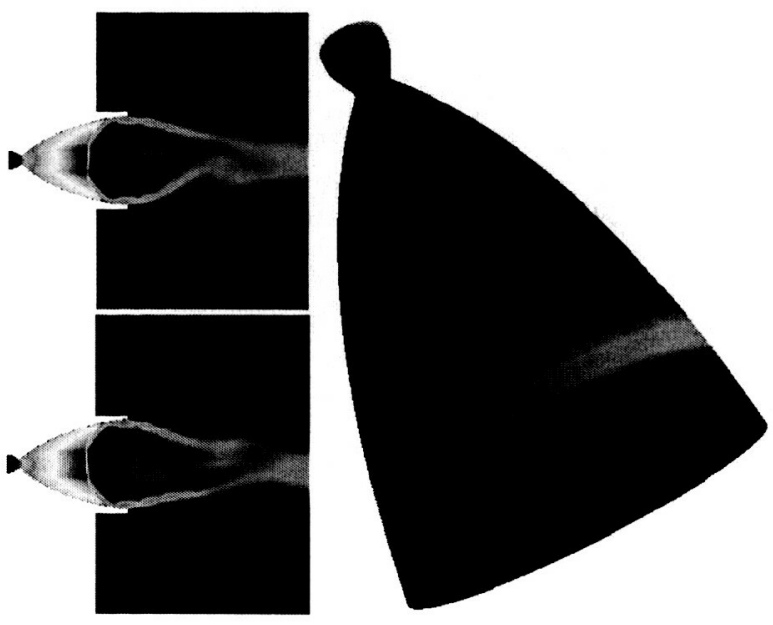

Fig. 11 Computed scalar contours for the cooled nozzle at $2.580 \mathrm{~s}$. Top left: Mach number contours on y-plane. Bottom left: Mach number contours on zplane. Right: wall $\mathrm{OH}$ concentration contours.

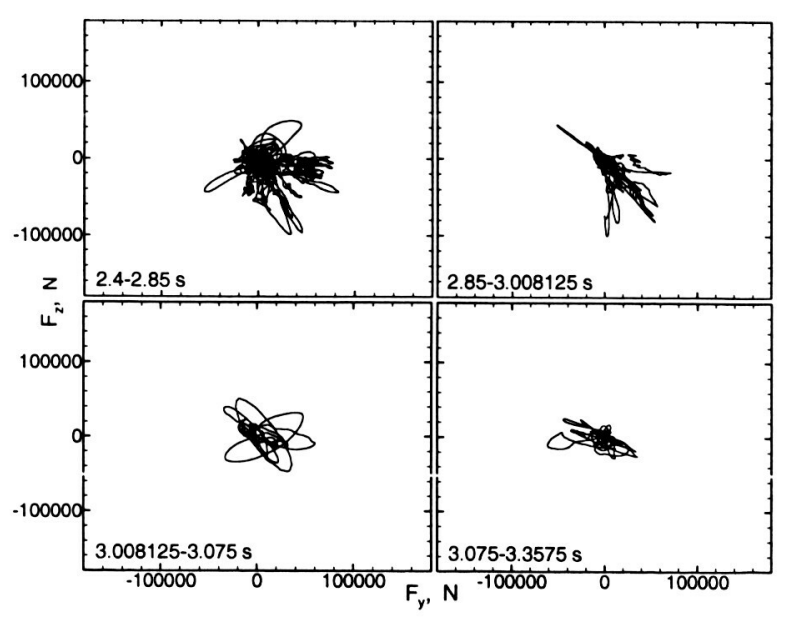

Fig. 12 Computed side force loci for the adiabatic nozzle. 


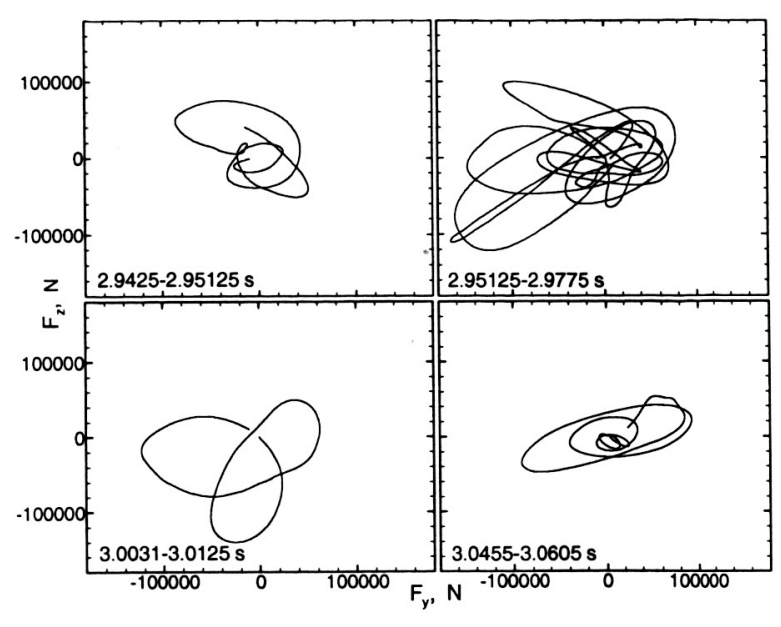

Fig. 13 Computed tangential force loci for the cooled nozzle.

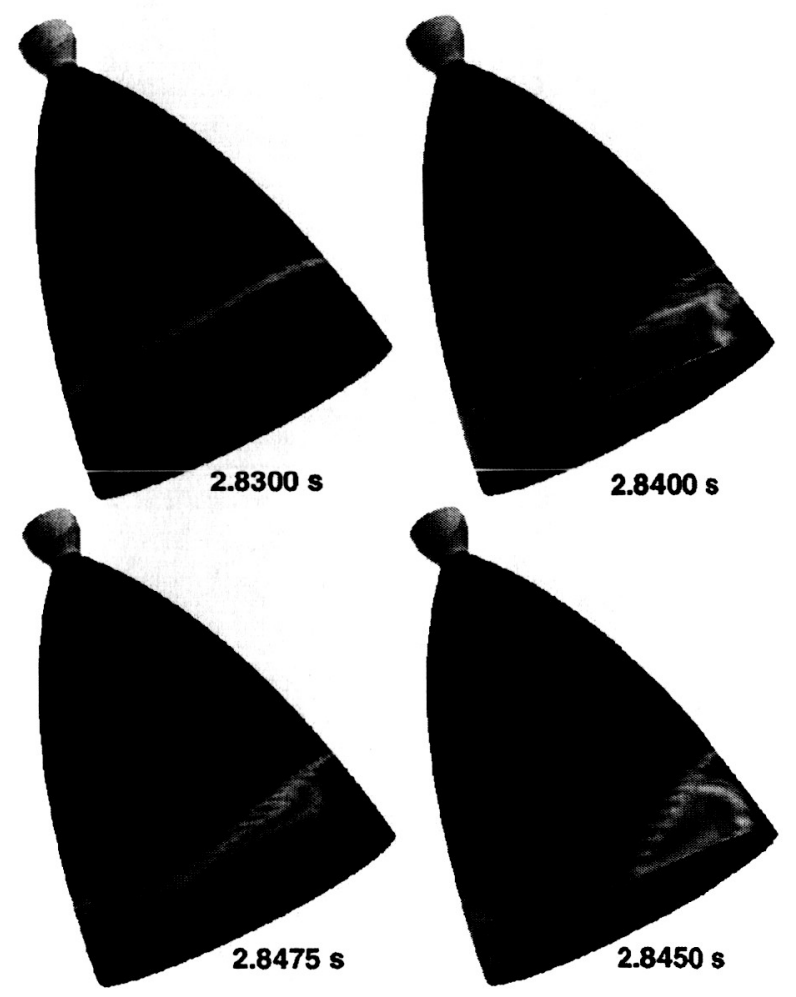

Fig. 14 Computed exhausting and receding wall $\mathrm{OH}$ contours for the adiabatic nozzle.

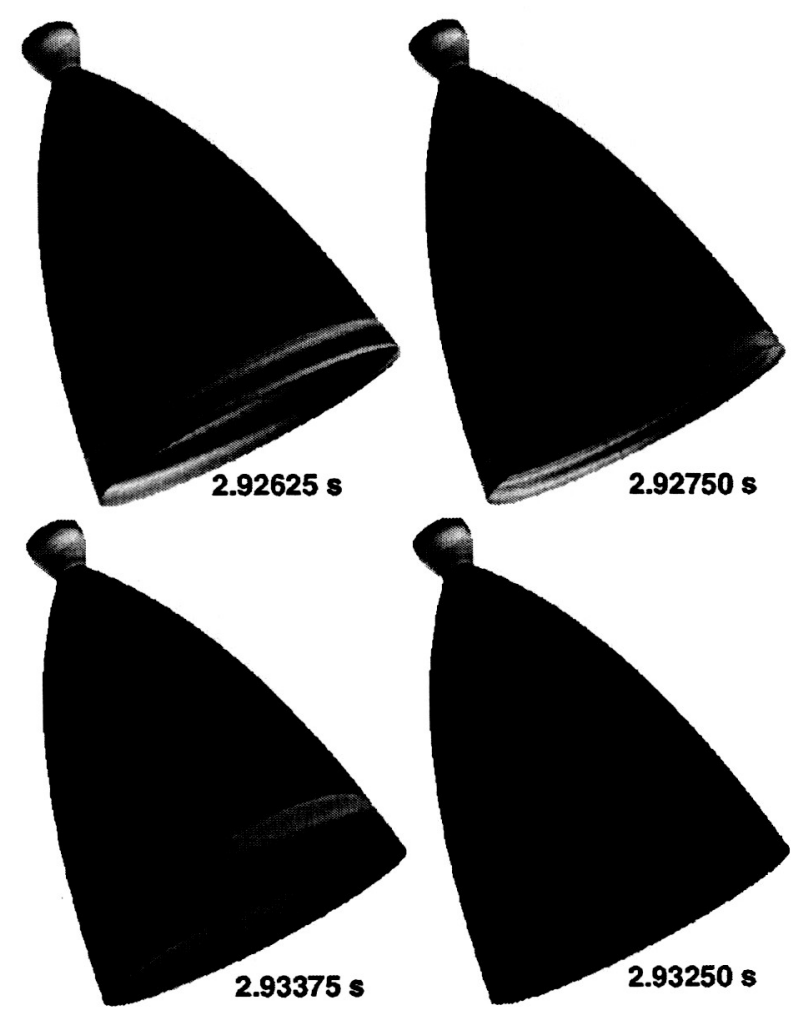

Fig. 15 Computed exhausting and receding wall $\mathrm{OH}$ contours for the cooled nozzle.

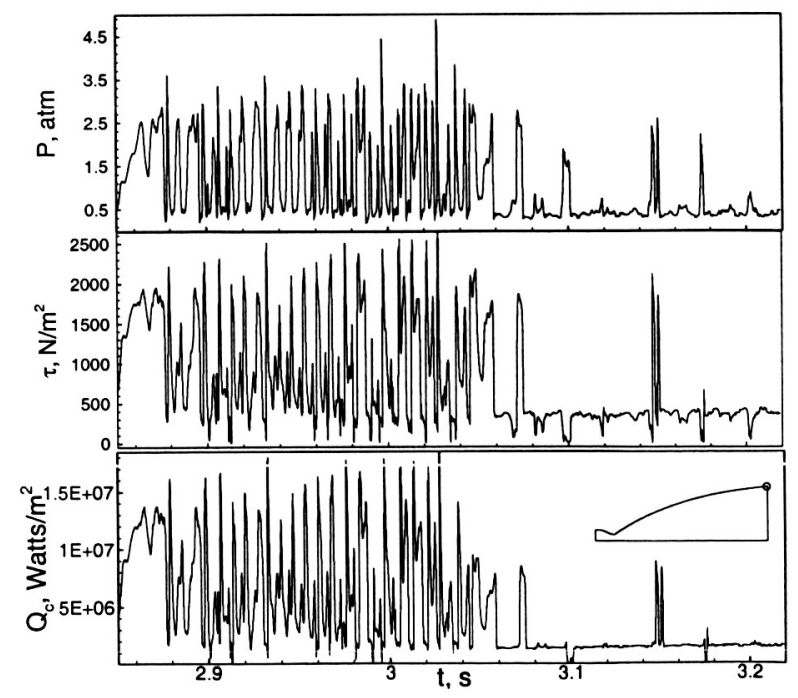

Fig. 16 Computed near lip wall pressure, shear stress, and heat flux histories for the cooled nozzle. 

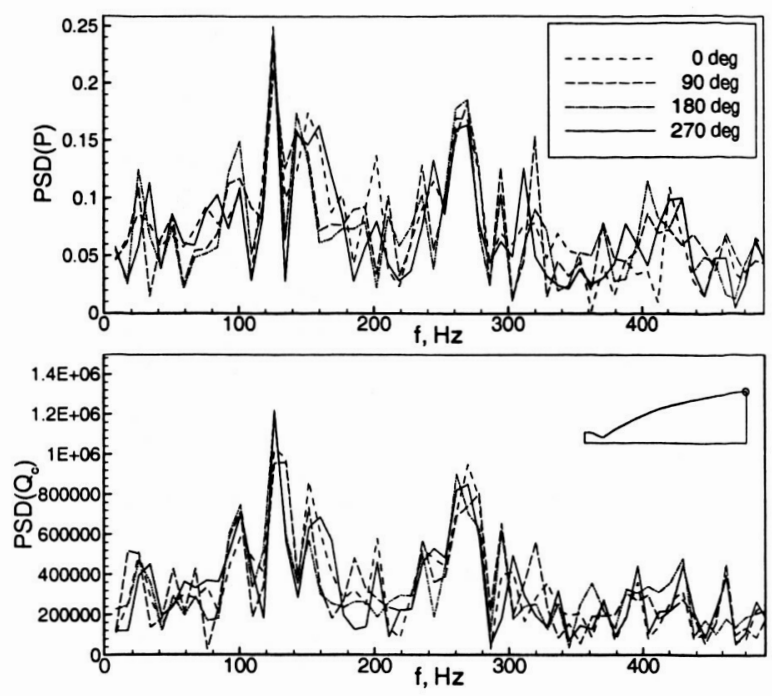

Fig. 17 Computed frequency domain for the cooled nozzle. 